\title{
REVIEW
}

\section{Global seabird bycatch in longline fisheries}

\author{
Orea R. J. Anderson ${ }^{1, *}$, Cleo J. Small ${ }^{1}$, John P. Croxall ${ }^{2}$, Euan K. Dunn ${ }^{1}$, \\ Benedict J. Sullivan ${ }^{1}$, Oliver Yates ${ }^{3}$, Andrew Black ${ }^{1,4}$ \\ ${ }^{1}$ BirdLife International Global Seabird Programme, Royal Society for the Protection of Birds, Sandy, \\ Bedfordshire, SG19 2DL, UK \\ ${ }^{2}$ BirdLife International Global Seabird Programme, BirdLife International, Wellbrook Court, Girton Road, \\ Cambridge, CB3 0NA, UK \\ ${ }^{3}$ BirdLife International Global Seabird Programme, Casilla 145, La Serena, Chile \\ ${ }^{4}$ Present address: Fisheries Department, Falkland Islands Government, PO Box 598, Stanley, FIQ 1ZZ, Falkland Islands
}

\begin{abstract}
Bycatch in longline fisheries is believed to govern the adverse conservation status of many seabird species, but no comprehensive global assessment has been undertaken. We reviewed the extent of seabird bycatch in all longline fisheries for which data are available. Despite the many inadequacies and assumptions contained therein, we estimated that at least 160000 (and potentially in excess of 320 000) seabirds are killed annually. Most frequently caught are albatrosses, petrels and shearwaters, with current levels of mortality liable to be unsustainable for some species and populations. Where realistic comparisons can be made, with data from the 1990s, there is evidence of substantially reduced bycatch in some key fisheries. Reductions stem from decreased fishing effort (especially in illegal, unreported and unregulated fishing in the Southern Ocean), and greater and more effective use of technical mitigation measures, notably in demersal fisheries. However, bycatch problems in other fisheries have also emerged. Current concerns include those with previously unidentified bycatch problems (e.g. Spanish Gran Sol demersal fleet) and those where bycatch was identified, but where persistent data gaps prevented adequate assessments of the scale of the impact (e.g. Nordic demersal fisheries). Future assessments will only achieve greater precision when minimum standards of data collection, reporting and analysis are implemented by longline fishing fleets and the relevant regional fishery management organisations. Those fisheries in which bycatch has been substantially reduced demonstrate that the problem of seabird bycatch could be reduced to negligible proportions by enforced implementation of appropriate best-practice mitigation devices and techniques.
\end{abstract}

KEY WORDS: Bycatch · Seabirds · Albatrosses $\cdot$ Global $\cdot$ Threats $\cdot$ Marine conservation

\section{INTRODUCTION}

Seventeen of the 22 species of albatross are threatened with extinction (IUCN 2010), with the key threat to most species recognised as incidental mortality (bycatch) associated with fisheries (Robertson \& Gales 1998). A further 7 species of petrel (Procellaria and Macronectes spp.) listed under the Agreement on the Conservation of Albatrosses and Petrels (ACAP), face similar threats (ACAP 2009). All of these procellariiform species are extremely wide-ranging, and their distributions overlap considerably with areas targeted by the world's fishing fleets (BirdLife International 2004). Albatrosses and petrels, along with other seabirds, come into conflict with fisheries when they forage behind vessels for bait and fish waste. The incidental mortality of seabirds on longlines was first reported from bird band recoveries in the early 1980s 
(Morant et al. 1983, Croxall et al. 1984), resulting from birds being caught and drowned on hooks while trying to snatch bait as the lines are set (Brothers 1991). More recently, the threat posed by trawl fisheries (whereby seabirds can become entangled in nets during shooting and hauling, or are killed by collision with warp cable as they forage behind the vessel) has also become apparent (Bartle 1991, Weimerskirch et al. 2000, Sullivan et al. 2006). Even in comparison with other seabird species, Procellariiformes are highly Kselected, so increases in adult mortality readily have significant adverse impacts on a population, several times more so than the loss of young birds (Croxall \& Rothery 1991, Véran et al. 2007, Igual et al. 2009). However, quantifying the scale of the problem is difficult due to the diverse and remote nature of many of the world's fisheries, the lack of systematic reporting, and the nature of seabird bycatch rates themselves, which can be highly variable. Nevertheless, several reviews have concluded that recent and/or current reported levels of seabird bycatch are demographically unsustainable for the populations involved (Croxall et al. 1998, Tuck et al. 2001, Arnold et al. 2006, Barbraud et al. 2009, Thompson et al. 2009, Rivalan et al. 2010).

Historically, fishermen have had mixed relationships with seabirds. Aggregations of birds have been used to indicate profitable fishing grounds (Crawford \& Shelton 1978), whereas catching non-target species, like seabirds, results in time lost through removing dead birds from hooks/nets, and fish catches foregone due to bait loss; these are detrimental to fishing activities and their economic efficiency. There is therefore, at least potentially, a common interest from conservation and fishery management perspectives alike in addressing this problem. In recent years, an effective response has emerged from the increasing variety and efficacy of technical measures designed to mitigate, and even eliminate, incidental catches of seabirds (e.g. Brothers et al. 1999, Bull 2007, FAO 2008, BirdLife International \& ACAP 2009). Despite this, there is considerable evidence that many fisheries do not use recommended best-practice mitigation measures (e.g. FAO 2008), which likely results in rates and levels of bycatch which may not have changed substantially since the problem was first identified.

Despite bycatch in fisheries being the main contributory factor influencing the adverse conservation status of many albatross and petrel species, there have been few attempts either to estimate the full magnitude of the problem, or to indicate which data may be sufficiently reliable to provide baselines for future comparisons. While several papers have reviewed seabird bycatch rates in longline fisheries in various regions (e.g. Brothers 1991, Dunn \& Steel 2001, Bugoni et al. 2008a, Rivera et al. 2008), only 1 attempt has been made to collate seabird bycatch data from longline fisheries on a global scale (Nel \& Taylor 2003). Furthermore, that study focused only on fisheries catching globally threatened seabirds (i.e. those listed on the IUCN Red List in 2000), and did not attempt to estimate an overall global bycatch level. In addition, most of the data available to Nel \& Taylor (2003) related to years prior to 2000. Considerable new data have been reported since then, and several new longline fisheries, thought likely to interact with seabirds, have commenced. All this makes a new and comprehensive review very timely. Such a review also needs to provide clear explanations of the interpretations and extrapolations inherent in working with sparse data provided in a wide variety of formats and with highly variable completeness and accuracy.

The present study aims to (1) review published and unpublished seabird bycatch data for longline fisheries worldwide and provide a comprehensive annotated archive of such information for future comparisons; (2) generate new estimates of seabird bycatch (including at a global scale) and compare these with previous reviews; (3) identify reasons for changes and emerging bycatch problems; (4) highlight continuing data gaps; and (5) indicate future challenges and provide recommendations for priority actions.

\section{METHODS}

Data on seabird bycatch. We reviewed the available published and unpublished literature on seabird and longline fishery interactions to obtain a comprehensive inventory of the most recent estimates (up to 2009) for seabird bycatch from longline fisheries around the world. All bird species caught on longlines were included in the review.

Where available, bycatch data from several years were combined in order to calculate an average number of seabirds caught per year in each fishery. Where changes in fisheries practice were obvious (e.g. implementation of new mitigation measures), data were selected to reflect the current situation, as far as data availability allowed.

In some cases, extracting relevant data was relatively straightforward. However, in many cases, assumptions, estimations and extrapolations were required. These are described in full in the Supplement (available at www.int-res.com/articles/suppl/ n014p091_supp.pdf) for each fishery examined (see Table 1). Two important examples are as follows. Firstly, for fisheries where seabird bycatch rate data (usually expressed as birds per unit effort, BPUE) were reported, but only for a sample of a fishery (a common 
event), these were scaled up to the level of the whole fishery using the relevant ratio of fishing effort. This assumes that bycatch rates are homogeneous across the areas and times in question. Secondly, for several key fishing fleets, no data are available on seabird bycatch. In those cases where bycatch rates were available from an analogous fishery (in terms of fishing method, target species and geographical area), and data were available on the magnitude and distribution of effort of the fishery in question, an extrapolation was made on this basis.

In order to provide some indication of the accuracy of the estimate of average number of birds killed in each fishery, a range around this figure was derived for as many fisheries as possible. Some sources included estimates of standard deviation or confidence limits around mean seabird bycatch rates. However, many did not or could not provide such estimates, and upper and lower ranges were more commonly available. Where range values were not provided in the source, these were calculated based on the upper and lower BPUE rates reported and the range in fishing effort across years (lower estimate $=$ lowest BPUE $\times$ lowest total fishing effort; upper estimate $=$ highest BPUE $\times$ highest total fishing effort). Where the required input variables were not available, no range was estimated. For estimating seabird bycatch associated with illegal, unreported and unregulated (IUU) fishing, data were taken from the review by the Marine Resources and Assessment Group (MRAG 2005).

Data reliability. We devised a measure to indicate how reliably the estimated values may reflect the true total seabird bycatch in each fishery. A scoring system was developed to account for the 3 main sources of error observed to occur within the datasets, each of which was scored as 'Poor', 'Medium' or 'Good'. The final classification of reliability was based on the lowest ranking in any of the 3 categories.

(1) Age of bycatch data: 1986-1994 = Poor, 1995-1999= Medium, 2000-2009 = Good.

(2) Source of bycatch data: all bycatch data derived from another fishery $=$ Poor; bycatch data partially derived from another fishery $=$ Medium; all data derived directly from the fishery in question $=$ Good.

(3) Accuracy: this reflects several different variables as follows: (i) the level of observer coverage from which a bycatch estimate was calculated $(<5 \%=$ Poor, $5-20 \%=$ Medium, $>20 \%=$ Good), where percent coverage is ideally defined as the proportion of hooks monitored relative to fleet fishing effort, but may also represent the proportion of sets or vessels monitored; (ii) the spatial and temporal extent of the observer coverage from which a bycatch estimate was calculated (low relative spatial and tem- poral coverage of observer effort $=$ Poor; low relative spatial or temporal coverage of observer effort = Medium; high relative spatial and temporal coverage of observer effort $=$ Good); (iii) the extent of spatial and/or temporal variability in the bycatch rates across the fishery, where known (i.e. high spatial and temporal variability $=$ Poor; high spatial or temporal variability $=$ Medium $;$ low spatial and temporal variability $=$ Good). Given that sources did not always report on all of these sub-categories, the overall score for 'Accuracy' was based on the subcategory into which the majority of variables (i) to (iii) fell. If only 2 sub-categories were reported on and their scores differed, an informed opinion was taken as to which category was most representative of the data source as a whole.

Comparison with previous reviews. Results were compared with the review by Nel \& Taylor (2003). Since that study focused only on fisheries catching threatened seabird species (predominantly albatrosses and petrels), it did not cover all the fisheries discussed in our review.

Data verification. Data were split by country and/or region and sent to relevant seabird and fishery experts for review (see 'Acknowledgements').

\section{RESULTS}

The results of the review of seabird bycatch in longline fisheries are shown in Table 1. Data were collected on 68 fisheries, and cover those operating in exclusive economic zones (EEZs) as well as the high seas. Extrapolated data are indicated in Table 1 in square brackets. Full notes on how each estimate was derived are provided in the Supplement. In relation to the data reliability score, 15 estimates were scored as having a 'Good' level of reliability, 23 were scored as 'Medium', and 30 were scored as 'Poor' (see Table S1 in the Supplement).

The sum of the estimated average number of seabirds killed in the 68 longline fisheries in Table 1 equals ca. 160000 seabirds killed globally each year in fisheries for which data are available. The 10 fleets with the highest levels of seabird bycatch are shown in Fig. 1 and include the Spanish hake fleet in the Gran Sol area, the Japanese pelagic tuna fleet in the North Pacific, the Namibian hake fleet and the Nordic demersal fleets. The data reliability score for 9 of the top 10 fleets was 'Poor'.

The sum of the upper ranges of the 68 fisheries equals ca. 320000 seabirds killed per year. This value is heavily influenced by the Norwegian demersal fleet (estimated average of 6514 birds caught each year, but with an upper range of 101380 birds $\mathrm{yr}^{-1}$ ). Other fleets 


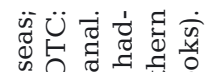

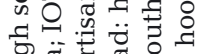

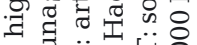

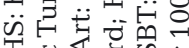
is

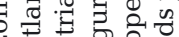

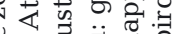
政记 政的

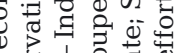

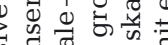

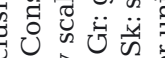

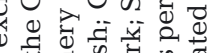
i 50 年 c.․ㅠ.

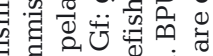

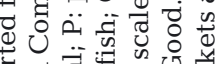

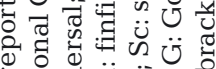

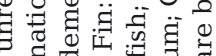
व

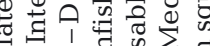

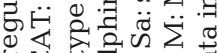

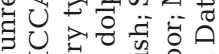
J S. 웅 .

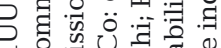

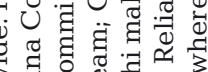

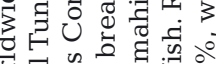

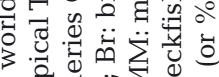
过然边 वै 包诸 o

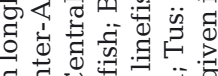
두월 उ

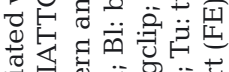

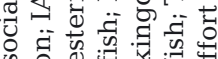

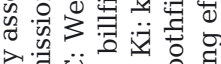

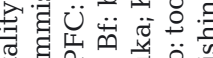
중 证了被 응을

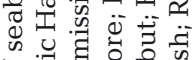

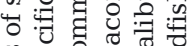
눙

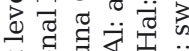

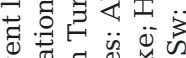

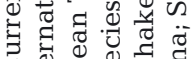
U.

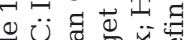

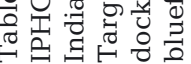

\begin{tabular}{|c|c|c|c|c|c|c|c|c|c|c|c|c|c|c|c|c|}
\hline әว.nоs & 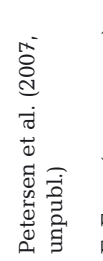 & 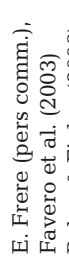 & 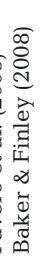 & 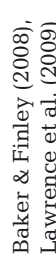 & & 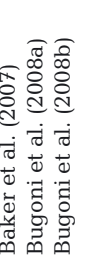 & 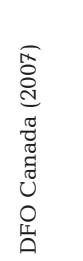 & & 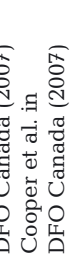 & 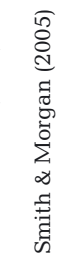 & 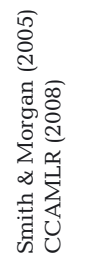 & 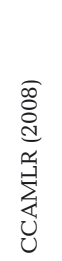 & 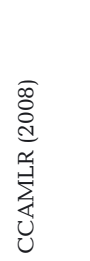 & 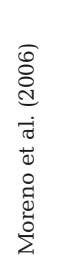 & 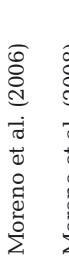 & 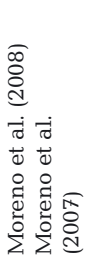 \\
\hline 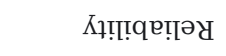 & a & $\Sigma$ & $\Sigma$ & u & $\Sigma$ & $\sum n$ & $\Sigma$ & $\Sigma$ & $\Sigma$ & $\Sigma$ & $\Sigma 0$ & 0 & v & v & a & $0 \Sigma$ \\
\hline $\begin{array}{r}\text { poẉəd } \\
\text { еұер чગұеəКя }\end{array}$ & 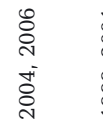 & 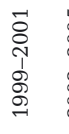 & 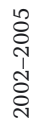 & 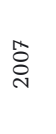 & ث্ं & 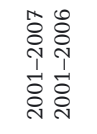 & $\overrightarrow{\mathrm{O}}$ & 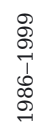 & $\begin{array}{l}\stackrel{8}{ } \\
\stackrel{9}{1} \\
0 \\
\stackrel{0}{9} \\
\stackrel{7}{7}\end{array}$ & 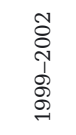 & 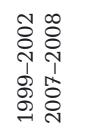 & 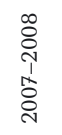 & $\begin{array}{l}\infty \\
\stackrel{े}{1} \\
1 \\
\stackrel{े}{0} \\
\text { ஸे }\end{array}$ & 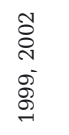 & & 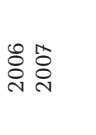 \\
\hline poțəd & 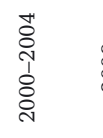 & 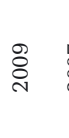 & 商 & 商 & $\stackrel{+}{\stackrel{4}{े}}$ & 芯 \& & $\overrightarrow{\stackrel{0}{0}}$ & & & 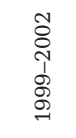 & 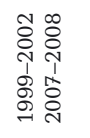 & 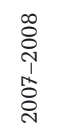 & 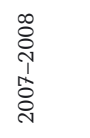 & ڤั่ & ઠ్రి & 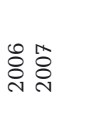 \\
\hline 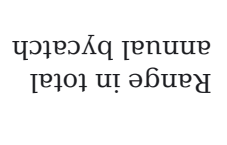 & & & & & & 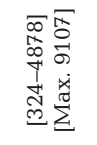 & 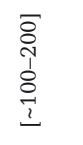 & & & & & & & & & 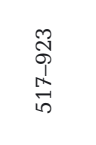 \\
\hline 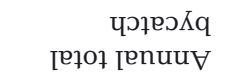 & 蛋 & $\underset{\substack{\infty \\
\stackrel{\infty}{C}}}{\infty}$ & 우 & ब্ণ & in & ت্ট్ & $\begin{array}{l}\overline{2} \\
\text { 垔 } \\
2\end{array}$ & 吕 & $\underset{7}{8}$ & in & No & $\vec{m}$ & $\underset{\sim}{\mathbb{J}}$ & 胥 & 禽 & $\circ \stackrel{\sqrt[n]{N}}{\underline{N}}$ \\
\hline घกdg uеәW & 要 & $\stackrel{\Delta}{\circ}$ & $\begin{array}{l}\bar{\Xi} \\
\dot{0}\end{array}$ & $\begin{array}{l}\infty \\
\text { In } \\
\text { Oे } \\
0\end{array}$ & $\stackrel{\overbrace{}}{0}$ & तึ? & $\begin{array}{l}\bar{N} \\
\text { 产 } \\
\text { อ }\end{array}$ & $\begin{array}{l}0 \\
\vdots \\
0\end{array}$ & 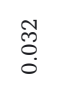 & $\begin{array}{l}\overrightarrow{7} \\
\text { 号 } \\
\dot{0}\end{array}$ & $\begin{array}{l}\text { 要。 } \\
\text { e }\end{array}$ & 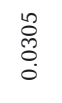 & $\begin{array}{l}2 \\
\infty \\
0 \\
0 \\
0\end{array}$ & $\stackrel{\%}{0}$ & 管 & 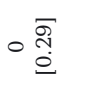 \\
\hline вн рәл.әәяфо & $\circ$ & & 总 & $\begin{array}{l}\text { 范 } \\
\text { on } \\
\text { 品 }\end{array}$ & ঃे & 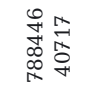 & $\stackrel{0}{0}_{1}^{\circ}$ & $\begin{array}{l}\stackrel{0}{0} \\
\stackrel{1}{1} \\
m\end{array}$ & 总 & $\stackrel{\circ}{\infty}$ & 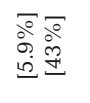 & 品 & $\begin{array}{l}\stackrel{0}{0} \\
\stackrel{i}{i}\end{array}$ & $\begin{array}{l}\widetilde{\tilde{ల}} \\
\text { ర్లె } \\
\end{array}$ & 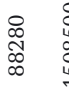 & 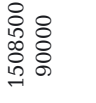 \\
\hline 코 u! әбиеप & & & & & & g & & & & & & & & & 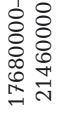 & \\
\hline घ日 иеәW & $\begin{array}{l}8 \\
8 \\
8 \\
0 \\
\infty\end{array}$ & $\begin{array}{l}\bar{\Xi} \\
\vdots \\
\vdots \\
\mathbb{J} \\
\text { I }\end{array}$ & $\begin{array}{l}0 \\
\vdots \\
\vdots \\
0 \\
0\end{array}$ & 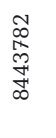 & 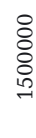 & 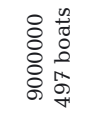 & & & & $\begin{array}{l}8 \\
\vdots \\
0 \\
0 \\
1 \\
1\end{array}$ & 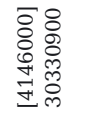 & 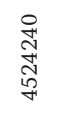 & 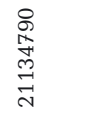 & 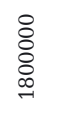 & 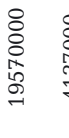 & 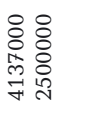 \\
\hline səțวəds łə6.ાе & 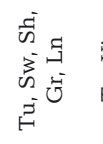 & $\begin{array}{l}7 \\
0 \\
0\end{array}$ & $\begin{array}{l}\text { की } \\
\text { फ̃ }\end{array}$ & $\begin{array}{l}\text { 岕 } \\
\vec{s} \\
\vec{F}\end{array}$ & $\begin{array}{l}\text { 荝 } \\
=\end{array}$ & H. & U & 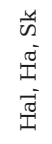 & 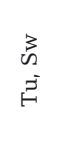 & 蛋 & $\ddot{\approx} \leftrightarrow$ & $\stackrel{\circ}{\leftrightarrow}$ & $\stackrel{\circ}{\bullet}$ & 胥 & $\stackrel{\circ}{\mapsto}$ & $\stackrel{ }{\circ} \mathrm{b}^{3}$ \\
\hline 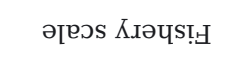 & $\Xi$ & 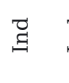 & 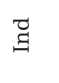 & $\Xi$ & $\Xi$ & 药 & $\Xi$ & $\Xi$ & $\widetilde{g}$ & $\Xi$ & $\exists \Xi$ & 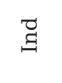 & $\Xi$ & 荌 & $\frac{7}{4}$ & 焉 \\
\hline 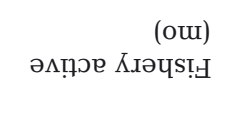 & 业 & ₹ & 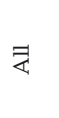 & $\exists$ & ঊ & 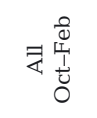 & & & & 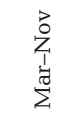 & $\nexists$ & 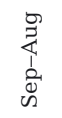 & 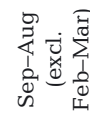 & & & 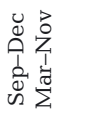 \\
\hline 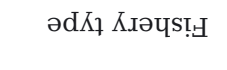 & a & ค & ค & a & & $D_{1} a_{1}$ & ค & ค & $a$ & A & ค & ค & a & & & $A{ }^{\prime}$ \\
\hline иотฺрวот & 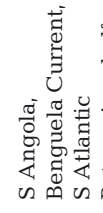 & $\begin{array}{l}\frac{w}{0} \\
\frac{\pi}{4} \\
0 \\
0 \\
0 \\
0 \\
\frac{\pi}{0} \\
0 \\
0\end{array}$ & 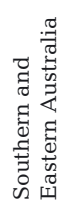 & 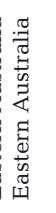 & 荇 & 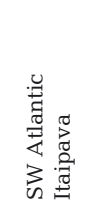 & 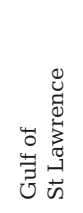 & 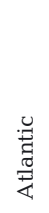 & 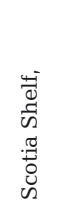 & 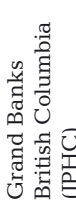 & 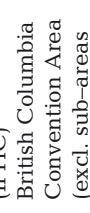 & 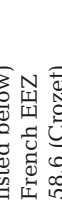 & 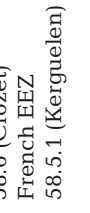 & 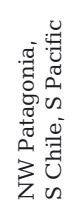 & 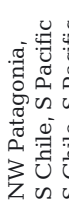 & 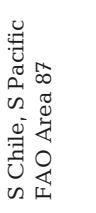 \\
\hline SH 으 Z큽 & 焗 & 恿 & 恿 & 触 & 裂 & 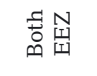 & 恿 & 恿 & 恿 & 恿 & 畄出 & 第 & 恿 & 恿 & 跡 & 舀营 \\
\hline K.дฺUnoว & 尊 & 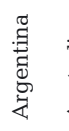 & 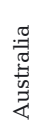 & 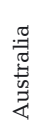 & ] & 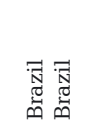 & $\begin{array}{l}\text { ] } \\
\text { ] } \\
\text { U }\end{array}$ & $\begin{array}{l}\text { J } \\
\pi \\
\tilde{J}\end{array}$ & $\begin{array}{l}\frac{\pi}{\pi} \\
\text { J } \\
\text { U }\end{array}$ & $\begin{array}{l}\text { 胥 } \\
\text { चี } \\
\text { Uี }\end{array}$ & 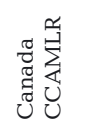 & 童 & 章 & चี & चี & : \\
\hline
\end{tabular}




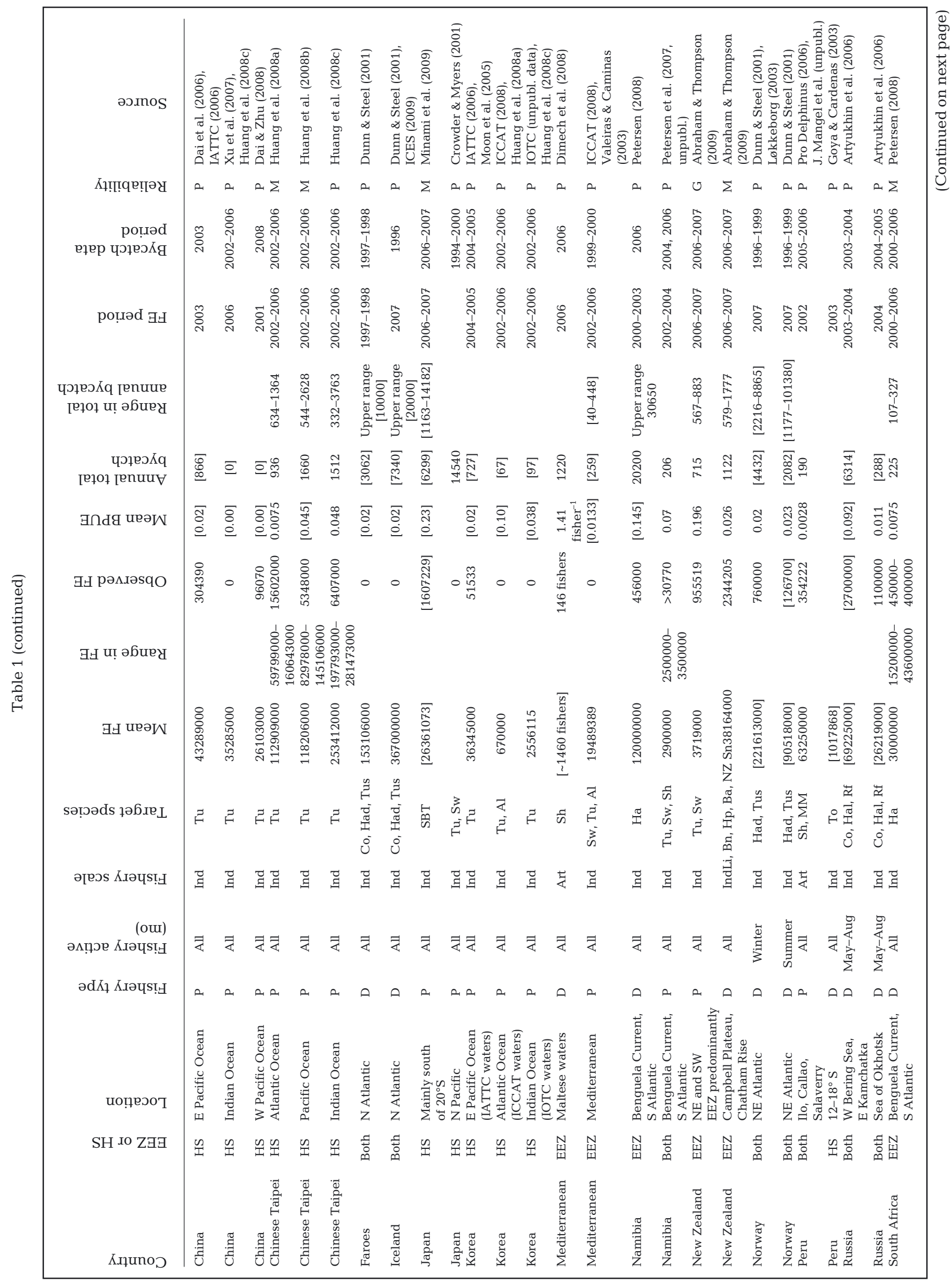




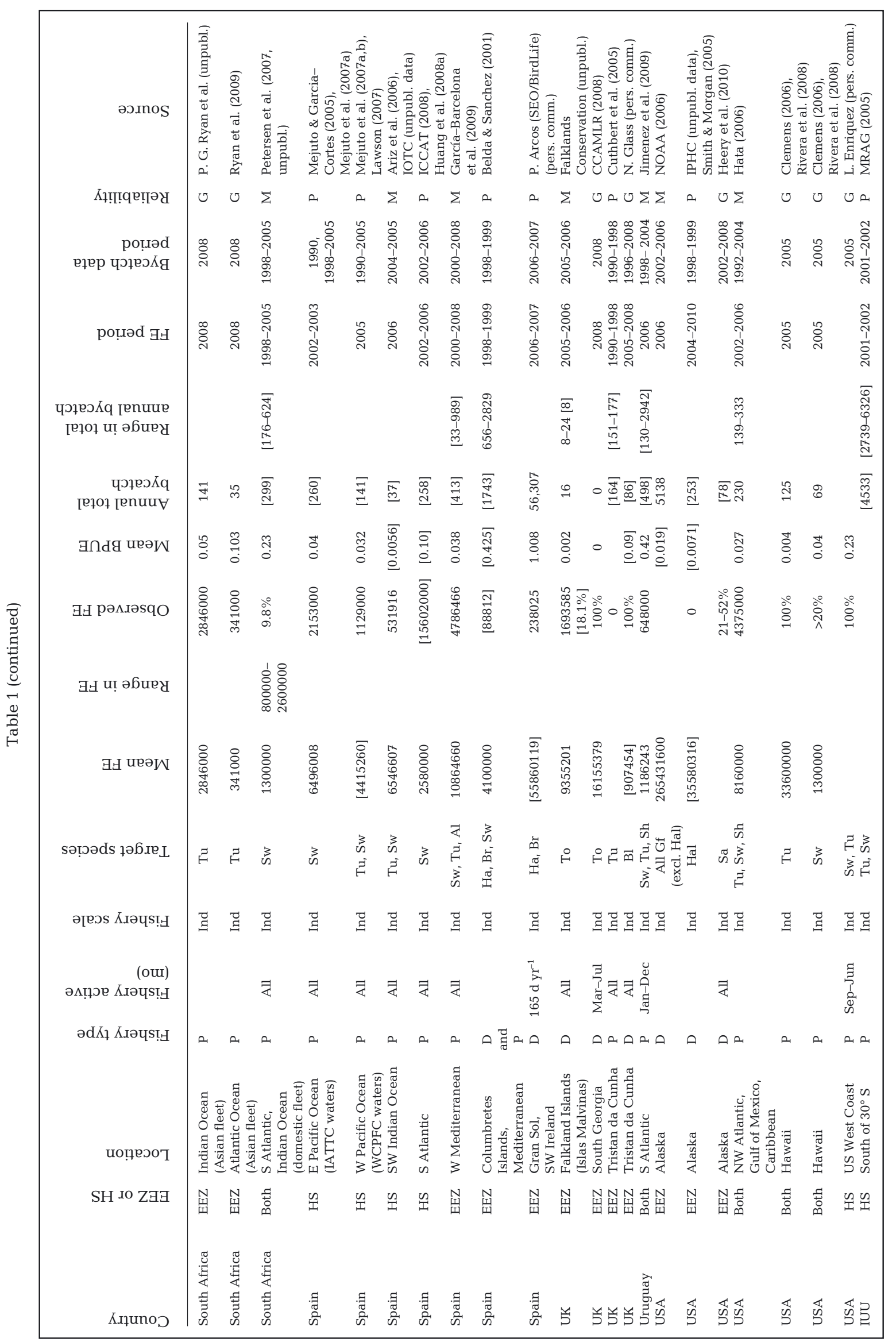




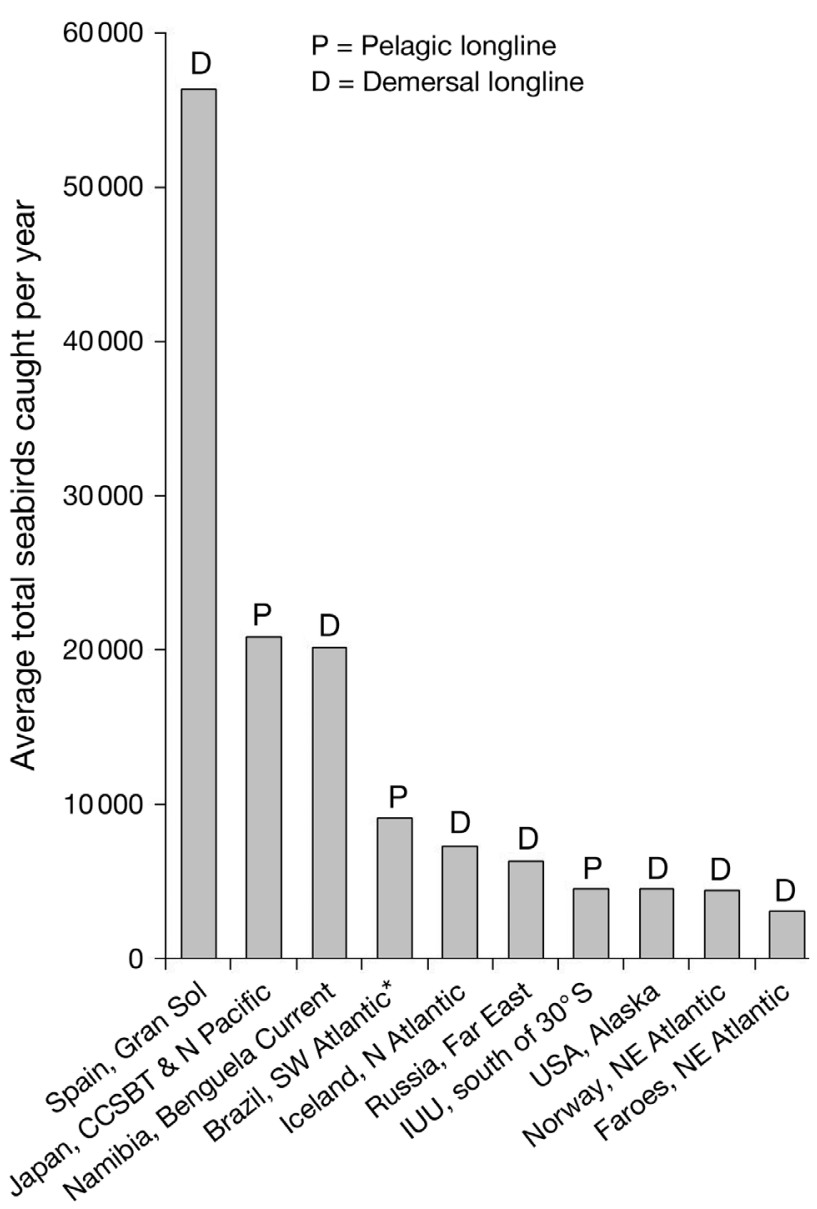

Fig. 1. Fishing fleets with the highest estimated average total numbers of seabirds killed per year. For further details on particular fleets, see the supplement at www.int-res.com/ articles/suppl/n014p091_supp.pdf. The asterisk indicates the maximum total seabirds caught per year. Spain: Gran Sol, Northeast Atlantic hake fishery; Japan: Commission for the Conservation of Southern Bluefin Tuna (CCSBT) and North Pacific estimates combined; Namibia: demersal hake fishery; Brazil: NW Atlantic pelagic fishery; Iceland: North Atlantic groundfish fishery; Russia: Far East groundfish fishery; IUU: illegal, unregulated and unreported pelagic longline activity south of $30^{\circ} \mathrm{S}$; USA: Alaskan demersal fishery (excluding halibut); Norway: NE Atlantic groundfish fishery; Faroes: NE Atlantic groundfish fishery

with large ranges in estimates include the Icelandic, Faroese, Russian and Namibian demersal fleets.

It was not possible to calculate a lower range of the global estimate of seabird bycatch because of the type of extrapolations required by the data. For example, if a fishery reported variable bycatch rates of 0.00 to 0.44 birds per 1000 hooks, the lower estimate would result in an estimate of 0 bycatch, regardless of any variation in fishing effort.

Sources varied considerably in terms of availability of species-specific bycatch data, and a global estimate of numbers caught by species or species group was not possible. However, the data available indicate that the vast majority of birds caught in longline fisheries were of the albatross (Diomedeidae), petrel and shearwater (Procellariidae) families, along with some species of gulls and terns (Laridae), gannets and boobies (Sulidae) and cormorants (Phalacrocoracidae). Data indicate that northern fulmar Fulmarus glacialis, great shearwater Puffinus gravis and white-chinned petrel Procellaria aequinoctialis are among those caught in the highest numbers, notably in the Spanish (Gran Sol), Nordic, Russian and Namibian demersal fisheries. For fleets operating south of $20^{\circ} \mathrm{S}$ and in the North Pacific, albatrosses and Procellaria petrels form a larger proportion of the bycatch. While these species may be being caught in lower numbers, the impact on their populations may be greater, as a result of their very low reproductive rates and, in most cases, relatively small population sizes. For some of the burrownesting petrels and shearwaters, such as the great shearwater, population trends are virtually unknown, and there may be an impact of bycatch on their populations that is currently unrecognised.

\section{DISCUSSION}

\section{Scale of global seabird bycatch in longline fisheries}

This review indicates that total annual seabird bycatch in longline fisheries is likely to be in excess of 160000 birds $\mathrm{yr}^{-1}$, and could be as high as 320000 birds $\mathrm{yr}^{-1}$, based on the average and upper range estimates, respectively, of the longline fisheries for which there are data. It should also be noted that the data reliability score (largely governed by levels of observer coverage) for 9 of the top 10 fleets was 'Poor', the exception being the Alaskan demersal groundfish fleet. It is uncertain whether this would result in bycatch estimates that were typically too low or too high. Nevertheless, the sum of the average estimates is very likely to be conservative, not only due to remaining data gaps (outlined below), but also because observed bycatch rates significantly underestimate actual total bycatch (Gales et al. 1998, Brothers 2008). Brothers (2008) reported only $50 \%$ of all birds observed caught during line setting were retrieved when the line was hauled aboard because of dead birds dropping off hooks prior to hauling.

The impact of this loss, on an annual basis, is impossible to assess without detailed species-specific population data. However, previous species-specific studies have assessed bycatch as a threat to relatively common species, such as black-browed albatross Thalassarche melanophrys and black-footed albatross Phoebastria nigripes (Arnold et al. 2006, Véran et al. 2007). For 
already highly globally threatened species, such as the Endangered Amsterdam albatross Diomedea amsterdamensis and the Critically Endangered Tristan albatross $D$. dabbenena, the impact of bycatch has been highlighted as a driving factor in population declines (Wanless et al. 2009, Rivalan et al. 2010). Greater understanding of species-specific impacts is vital. As an example, the Uruguayan pelagic longline fishery catches many fewer birds than the Spanish Gran Sol fishery. However, albatrosses make up $>80 \%$ of all seabird bycatch in Uruguay (Jimenez et al. 2009). Many of these birds are wandering albatrosses $D$. exulans from South Georgia, and these losses alone are sufficient to account for much of the continuing (and recently increased) pattern of decline seen in South Georgia wandering albatross populations in recent decades (Croxall et al. 1998, Tuck et al. 2001, Poncet et al. 2006, Phillips et al. 2010).

\section{Comparisons with previous estimates}

Full details of comparisons with previous estimates from Nel \& Taylor (2003) are provided in Table S2 in the Supplement, and key elements are summarised in Table 2. The comparison highlights changes that have occurred in some fisheries between the mid-1990s and mid-2000s. Where there have been decreases in total numbers of birds caught since Nel \& Taylor (2003), the causative factors can be categorised as follows (these categories are also used in Table 2):

(1) Greater or more effective use of mitigation measures;

(2) Changes in fishing practices, particularly using gear or methods less likely to catch seabirds;

(3) Reduction in fishing effort within a particular fleet;

(4) Collapse of a particular fishery as a result of overfishing of target species;

(5) New data available with various and/or unidentifiable causative factors for decrease in bycatch (e.g. varying sample sizes, locations, methodologies).

Where there have been increases in total numbers of birds caught by a fishery, the causative factors can be categorised as follows (categories used in Table 2):

(6) No entry for the fishery in Nel \& Taylor (2003) because of an unknown bycatch problem, but new data now available;

(7) No estimate for the fishery in Nel \& Taylor (2003) because of a lack of data reportage, but new data now available;

(8) Increase in fishing effort within a particular fleet.

The main fleets for which there have been major decreases in bycatch between the 2 review periods include the following.
Demersal longline fleets operating in Commission for the Conservation of Antarctic Marine Living Resources (CCAMLR) waters. Seabird bycatch in the CCAMLR region has decreased substantially in recent years, predominantly due to a decrease in IUU demersal longline activity, which has in turn stemmed from increased enforcement and international policing of the region. The drop in IUU longline activity has led to a reduction in bycatch of ca. 67000 birds $\mathrm{yr}^{-1}$ since the early 2000s. Meanwhile, bycatch in the regulated fisheries has also been substantially reduced, due to the implementation of a comprehensive suite of mitigation measures, including closed seasons (Croxall 2008).

New Zealand demersal ling fleet, South Africa licensed Asian pelagic tuna fleet and the US Alaskan demersal groundfish and Hawaiian pelagic tuna and swordfish fleet. These countries have implemented FAO National Plans of Action (NPoA-Seabirds) (Anon. 2001, 2004, 2008), which require the use of seabird bycatch mitigation measures backed up by observer programs. Comparisons with previous figures for the fleets of these 3 countries indicate a reduction in estimated bycatch of ca. 4000, 17000 and 15000 birds yr $^{-1}$, respectively.

Japanese distant water southern bluefin tuna Thunnus maccoyi fleet. The latest estimates point to a potential reduction in total seabird bycatch of ca. 11000 birds $\mathrm{yr}^{-1}$ since estimates from the late 1990s and early 2000s. This stems from (1) a reduction in reported fishing effort from 43 million to 26 million hooks $\mathrm{yr}^{-1}$ and (2) a reduction in reported average bycatch rates from to 0.37 to 0.23 birds per 1000 hooks. However, the uncertainty surrounding the new bycatch estimate remains high, with an upper range of ca. 14182 birds $\mathrm{yr}^{-1}$ (Minami et al. 2009).

Uruguayan pelagic industrial fleet for tuna, swordfish and sharks. The total fishing effort for this fleet appears to have declined considerably since the previous estimate (20 million hooks previously, 1.2 million hooks currently). The drop in estimated bycatch from ca. 6000 to ca. 500 birds $\mathrm{yr}^{-1}$ reflects this reduction in effort. The upper range on this estimate remains at ca. 3000 birds $\mathrm{yr}^{-1}$.

Brazilian demersal hake and pelagic tuna and swordfish fleets. The collapse of the demersal hake fishery has led to a reduction of ca. 4000 birds killed each year. In the tuna fishery, the estimated number of birds killed per year has also reduced by ca. 4000, in this case as a result of new data and the implementation of mitigation measures. It should be noted that seabird bycatch in the Itaipava fleets is an issue that has emerged since previous estimates, and could amount to up to ca. 10000 birds killed each year.

These results suggest an overall decrease in seabird bycatch of ca. 127500 birds killed each year in the fish- 
Table 2. Current and previous (Nel \& Taylor 2003) estimates of numbers of seabirds killed per year in longline fisheries, with likely causes of change between the 2 periods. CCAMLR: Commission for the Conservation of Antarctic Marine Living Resources; IUU: illegal, unregulated, unreported fishing; IATTC: Inter-American Tropical Tuna Commission; EEZ: exclusive economic zone; WCPFC: Western and Central Pacific Fisheries Commission; UKOT: UK Overseas Territories. Fishery type-D: demersal; P: pelagic. NA: not available. HIMI: Heard and Macquarie Islands. Figures in square brackets are extrapolated from other data. Categories for 'Reason for change' are listed in the 'Discussion'. For further information see Table S2 in the Supplement at www.int-res.com/articles/suppl/n014p091_supp.pdf

\begin{tabular}{|c|c|c|c|c|c|}
\hline Country & Location & $\begin{array}{c}\text { Fishery } \\
\text { type }\end{array}$ & $\begin{array}{l}\text { Previous } \\
\text { estimate }\end{array}$ & $\begin{array}{l}\text { Current } \\
\text { estimate }\end{array}$ & $\begin{array}{l}\text { Reason for } \\
\text { change }\end{array}$ \\
\hline Angola & S Angola, Benguela current, S Atlantic & $\mathrm{P}$ & NA & 245 & 6 \\
\hline Argentina & Patagonian shelf & $\mathrm{D}$ & 1160 & {$[58]$} & 3 \\
\hline Australia & S and E Australia & $\mathrm{D}$ & NA & 10 & 7 \\
\hline Australia & E Australia & $\mathrm{P}$ & NA & [209] & 7 \\
\hline Australia & W Australia & $\mathrm{P}$ & NA & {$[30]$} & 7 \\
\hline Brazil & SW Atlantic Ocean & $\mathrm{P}$ & 6656 & [2061] & 1,5 \\
\hline Brazil & Itaipava & $\mathrm{P}$ & NA & [Max. 9107] & 6 \\
\hline Brazil & SW Atlantic & $\mathrm{D}$ & 4214 & 0 & 4,5 \\
\hline Canada & Gulf of St. Lawrence & $\mathrm{D}$ & NA & {$[70-327]$} & 6 \\
\hline Canada & Atlantic & $\mathrm{D}$ & NA & 500 & 6 \\
\hline Canada & Scotia Shelf, Grand Banks & $\mathrm{P}$ & NA & 1400 & 6 \\
\hline Canada & Pacific & $\mathrm{D}$ & NA & 54 & 7 \\
\hline Canada & Pacific & $\mathrm{D}$ & NA & 72 & 6 \\
\hline CCAMLR & Convention Area (excl. sub-areas listed below) & $\mathrm{D}$ & 14050 & 0 & 1,3 \\
\hline CCAMLR & Sub-areas 58.6 and 58.7 (Crozet \& Prince Edward Islands) & $\mathrm{D}$ & $10583^{\mathrm{a}}$ & 131 & 1,3 \\
\hline CCAMLR & Sub-areas 58.5.1 and 58.5.2 (Kerguelen and HIMI) & $\mathrm{D}$ & $43597^{\mathrm{a}}$ & 1224 & 1,3 \\
\hline Chile & NW Patagonian region, S Chile, S Pacific Ocean & $\mathrm{D}$ & NA & [54] & 7 \\
\hline Chile & NW Patagonian region, S Chile, S Pacific Ocean & $\mathrm{D}$ & NA & 437 & 7 \\
\hline Chile & S Chile, S Pacific & $\mathrm{D}$ & NA & 0 & 7 \\
\hline Chile & FAO Area 87 & $\mathrm{P}$ & NA & $517-923$ & 7 \\
\hline China & E Pacific Ocean & $\mathrm{P}$ & NA & [866] & 6 \\
\hline China & Indian Ocean & $\mathrm{P}$ & NA & [0] & 6 \\
\hline China & W Pacific Ocean & $\mathrm{P}$ & NA & [0] & 6 \\
\hline Chinese Taipei & Atlantic Ocean & $\mathrm{P}$ & NA & 936 & 6 \\
\hline Chinese Taipei & Pacific Ocean & $\mathrm{P}$ & 2945 & 1660 & 5 \\
\hline Chinese Taipei & Indian Ocean & $\mathrm{P}$ & NA & 1512 & 6 \\
\hline Japan & Mainly south of $20^{\circ} \mathrm{S}$ & $\mathrm{P}$ & {$[17242]$} & [6299] & 3,5 \\
\hline Japan & North Pacific Ocean & $\mathrm{P}$ & 14540 & 14540 & - \\
\hline Korea & East Pacific Ocean (IATTC waters) & $\mathrm{P}$ & NA & {$[727]$} & 6 \\
\hline Korea & Indian Ocean, south of $20^{\circ} \mathrm{S}$ & $\mathrm{P}$ & NA & [97] & 6 \\
\hline Korea & Atlantic Ocean & $\mathrm{P}$ & NA & [67] & 6 \\
\hline Mediterranean & Maltese waters & $\mathrm{D}$ & NA & 1220 & 6 \\
\hline Mediterranean & Mediterranean & $\mathrm{P}$ & NA & {$[259]$} & 6 \\
\hline Namibia & Benguela current, S Atlantic & $\mathrm{D}$ & NA & 20,200 & 6 \\
\hline Namibia & Benguela current, S Atlantic & $\mathrm{P}$ & NA & 206 & 6 \\
\hline New Zealand & NE and SW EEZ predominantly & $\mathrm{P}$ & NA & 715 & 7 \\
\hline New Zealand & Campbell Plateau, Chatham Rise & $\mathrm{D}$ & 4958 & 1122 & 1,8 \\
\hline Peru & Ilo, Callao, Salaverry & $\mathrm{P}$ & 3990 & 190 & 5 \\
\hline Peru & $12-18^{\circ} \mathrm{S}$ Pacific Ocean & $\mathrm{D}$ & NA & NA & 6 \\
\hline Russia & W Bering Sea, E Kamchatcka (Pacific) & $\mathrm{D}$ & NA & [6334] & 6 \\
\hline Russia & Sea of Okhotsk & $\mathrm{D}$ & NA & [288] & 6 \\
\hline South Africa & Benguela current, S Atlantic Ocean & $\mathrm{D}$ & NA & 225 & 6 \\
\hline South Africa & Indian Ocean (Asian fleet) & $\mathrm{P}$ & {$[17427]$} & 141 & $1,3,5$ \\
\hline South Africa & Atlantic Ocean (Asian fleet) & $\mathrm{P}$ & as above & 35 & $1,3,5$ \\
\hline South Africa & S Atlantic, Indian Ocean (Domestic fleet) & $\mathrm{P}$ & {$[354]$} & [299] & 1 \\
\hline Spain & East Pacific Ocean (IATTC waters) & $\mathrm{P}$ & NA & [260] & 6 \\
\hline Spain & West Pacific Ocean (WCPFC waters) & $\mathrm{P}$ & NA & [141] & 6 \\
\hline Spain & SW Indian Ocean & $\mathrm{P}$ & NA & [37] & 6 \\
\hline Spain & S Atlantic & $\mathrm{P}$ & NA & [258] & 6 \\
\hline Spain & W Mediterranean & $\mathrm{P}$ & NA & [413] & 6 \\
\hline Spain & Columbretes Islands, Mediterranean & $\mathrm{D}, \mathrm{P}$ & NA & [1743] & 6 \\
\hline Spain & Gran Sol, SW Ireland & $\mathrm{D}$ & NA & 56307 & 6 \\
\hline UK & Falkland Islands (Islas Malvinas) & $\mathrm{D}$ & 40 & {$[16]$} & 1,3 \\
\hline UK & South Georgia & $\mathrm{D}$ & $66^{\mathrm{a}}$ & 0 & $1,2,3$ \\
\hline UK & Tristan da Cunha, UKOT & $\mathrm{P}$ & NA & {$[164]$} & 6 \\
\hline UK & Tristan da Cunha, UKOT & $\mathrm{D}$ & NA & [86] & 6 \\
\hline
\end{tabular}


Table 2 (continued)

\begin{tabular}{|c|c|c|c|c|c|}
\hline Country & Location & $\begin{array}{c}\text { Fishery } \\
\text { type }\end{array}$ & $\begin{array}{l}\text { Previous } \\
\text { estimate }\end{array}$ & $\begin{array}{l}\text { Current } \\
\text { estimate }\end{array}$ & $\begin{array}{c}\text { Reason for } \\
\text { change }\end{array}$ \\
\hline Uruguay & S Atlantic & $\mathrm{P}$ & [6000] & [498] & 3,5 \\
\hline USA & Alaska (groundfish) & $\mathrm{D}$ & 16800 & 5138 & 1,5 \\
\hline USA & Alaska (rockfish) & $\mathrm{D}$ & as above & {$[78]$} & 1,5 \\
\hline USA & NW Atlantic, Gulf of Mexico, Caribbean & $\mathrm{P}$ & NA & 230 & 6 \\
\hline USA & Hawaii (tuna) & $\mathrm{P}$ & 3268 & 125 & 1,5 \\
\hline USA & Hawaii (swordfish) & $\mathrm{P}$ & as above & 69 & 1,5 \\
\hline IUU & South of $30^{\circ} \mathrm{S}$ & $\mathrm{P}$ & NA & [4533] & 6 \\
\hline
\end{tabular}

eries listed above, driven both by the use of mitigation measures, changing fishing practices and reduction of fishing effort (Table 2). All of these fisheries overlap with albatross distributions, indicating potentially important reductions in the numbers of albatrosses being caught. However, for some species there remains the possibility that part of any decrease actually reflects diminished populations available to interact with longline fisheries, following a decade or more of unsustainable levels of bycatch. In other words, the proportion of a population being killed as bycatch may remain the same, despite reductions in the total numbers of birds being killed.

\section{Emerging bycatch problems}

Progress made towards seabird bycatch reduction in the fisheries listed above is tempered by new information concerning significant bycatch in other fleets. New bycatch data account for ca. 90730 birds killed each year, all of which was previously unknown and/or unaccounted for in the review by Nel \& Taylor (2003), and include the following.

Spanish demersal longline fishery (Gran Sol, North Atlantic). The highest estimated average annual mortality of seabirds in any fishery exists in the Spanish demersal longline fishery operating on Gran Sol, North-East Atlantic (ca. 56000 birds $\mathrm{yr}^{-1}$ ), based on data collected in 2006 to 2007 . The majority of birds caught in this fishery are great shearwaters, a species not currently believed to have a declining global population (though few, if any, relevant data exist). Nevertheless, the sheer scale of the numbers caught is cause for concern. Further study is required to verify that the bycatch rate is routinely of this magnitude.

Namibian fleets. Seabird bycatch in Namibia did not feature in previous reviews due to an absence of data. The limited information now available points to large numbers of birds being caught by the demersal fleet.
Petersen (2008) reported a potential bycatch estimate of ca. 20200 birds $\mathrm{yr}^{-1}$. While the majority of this bycatch is thought to be petrels, albatrosses contribute ca. 600 ind. $\mathrm{yr}^{-1}$ to the total, which includes the Critically Endangered Tristan albatross.

Russian Far East demersal longline fishery. Seabird bycatch data from the Russian industrial demersal fleets operating in the Kamchatka region and the Sea of Okhotsk have only become available in recent years. Artyukhin et al. (2006) estimated that ca. 10000 seabirds were killed in the fishery in 2003 and ca. 2745 seabirds in 2004, resulting in an annual average of ca. 6500 birds killed per year. Species caught include northern fulmar, slaty-backed gull Larus schistisagus and short-tailed shearwater Puffinus tenuirostris. No mitigation measures were reported in use, and bycatch rates varied considerably, both spatially and temporally. This variation may stem from low levels of observer coverage ( $3 \%$ of total effort in 2003), but could also relate to inter-annual variations in the distributions of seabirds and fishing effort.

\section{Continuing data gaps}

Globally, there remain many longline fisheries with insufficient data to assess seabird bycatch. Major data gaps remain for artisanal fleets, such as those in the Mediterranean, West Africa and Northwest Pacific, and many industrial fleets. Some of the main data gaps, for those fleets that have high spatial overlap with vulnerable seabird species, are summarised below.

North-East Atlantic demersal longline fleets. The large uncertainty over seabird (mainly northern fulmar) bycatch levels associated with Norwegian, Icelandic and Faroese demersal fleets in the North Atlantic reflects the fact that the bycatch estimates for all 3 fisheries are based on data collected from the Norwegian fleet over a decade ago. With upper range estimates of annual bycatch nearing 140000 birds for the 3 
fleets combined, it is essential that these fleets be adequately assessed for current bycatch rates, and for true impacts on the relevant seabird populations in the North Atlantic to be characterised. No estimates are currently available for demersal fleets from Greenland or the Barents Sea.

Asian distant water pelagic longline fleets. Significant uncertainty over longline-related seabird bycatch continues in relation to the large Asian distant water pelagic fleets. Data were available from Chinese Taipei fleets fishing in the Atlantic, Indian and Pacific Oceans, but few data were available for the Japanese fleet outside those reported to the Commission for the Conservation of Southern Bluefin Tuna (CCSBT); few data are also available from the Korean and Chinese fleets (see the Supplement).

Mediterranean fleets. Cooper et al. (2003) highlighted the lack of seabird bycatch data available for the Mediterranean. The only rigorous scientific investigations to date have come from Spanish waters in the western Mediterranean (e.g. García-Barcelona et al. 2009). Elsewhere, there are thousands of vessels, mostly artisanal, fishing within the region, yet very little is known of their impacts on seabirds or other vulnerable species. The limited data available indicate that several species of shearwater, namely Balearic Puffinus mauretanicus, Yelkouan P. yelkouan and Cory's Calonectris diomedea, are caught in numbers that may prove to be unsustainable for the potential source populations concerned (Igual et al. 2009). The European Commission has recently taken steps towards an EU Plan of ActionSeabirds to reduce the incidental catch of seabirds in longline and other fisheries, which may stimulate further study of Mediterranean and other poorly documented fisheries within European waters, and may also recommend measures to curb the impact of distant water fleets registered to European states.

Humboldt Current fleets. The Humboldt Current is a particularly important over-wintering ground for several species of albatross that breed in New Zealand, as well as being a key part of the foraging range of the Critically Endangered waved albatross Phoebastria irrorata and of several other globally threatened species of Procellaria petrels from New Zealand and South Georgia. Bycatch and directed take (intentional hunting) are known to occur in this region, but few data are available to quantify the scale of the problem (Pro Delphinus 2006, Ayala et al. 2008).

IUU fisheries bycatch. Catch rates associated with IUU activity are inherently difficult to assess. Estimates for seabird bycatch in IUU longline fisheries in this review are only for latitudes south of $30^{\circ} \mathrm{S}$ (MRAG 2005). Although most threatened albatross and Procellaria petrel species occur south of $30^{\circ} \mathrm{S}$, the potential that significant levels of seabird bycatch continue to occur in IUU fisheries north of this latitude cannot be discounted while such substantial data gaps remain.

\section{Future challenges to improving bycatch estimates}

Our review highlights 2 key issues that must be addressed before global estimates of seabird bycatch can be further improved: the lack of observer programs in certain key fleets and/or inadequate spatial and temporal coverage by onboard observer programs; and the need for standardisation in seabird bycatch data collection and reporting.

Increasing coverage by onboard observer programs. A significant number of longline fisheries remain for which no, or very limited, seabird bycatch data are available. Within those fleets, the number of hauled hooks observed is frequently $<1 \%$ of total fishing effort, and such data as are collected commonly have inadequate spatial and/or temporal coverage of the fleet. To accurately monitor rates of seabird bycatch, observation of $\geq 20 \%$ or more of the hooks may be required (Ashford 2002, Lawson 2006), though in many cases having representative coverage of $>5 \%$ would be a significant improvement. Sampling strategies must ensure that the observed hooks are spatially and temporally representative of the fishery.

Data collection, analysis and reporting standards. Inconsistencies in the formats of data reported currently hamper our ability to compare seabird bycatch rates between fisheries or over time. Best practice methods for collecting bycatch data have been elaborated (e.g. Dietrich et al. 2007), and establishment of agreed minimum standards for collecting and reporting bycatch data is vital to assist future assessment and mitigation efforts on the catch of non-target species (not just of seabirds), and to ensure transparency for all stakeholders. Based on this review, to allow comparison, reporting should include, at a minimum:

(1) The number of hauled hooks observed per year within the fleet and the proportion of total fishing effort that this represents;

(2) Information on the spatial and temporal distribution of observer effort within the fishery;

(3) The number of birds observed caught (including species identification and status, i.e. dead or alive) and a bycatch rate per thousand hooks;

(4) An estimate of total seabird bycatch along with a stated methodology as to how figures were derived.

\section{CONCLUSIONS}

We estimate that at least 160000 birds (with an upper range of 320000 birds) are killed each year in global longline fisheries. However, for almost all cur- 
rent estimates, the absolute levels of seabird bycatch will be substantially higher (by as much as $50 \%$ ) due to birds killed being unobserved or under-reported. Taking this and other identified data gaps into account, the true global level is likely to be substantially higher.

For those fleets for which seabird bycatch data have been reported, the fisheries with the highest levels of seabird mortality are the demersal fleets of Spain, Namibia, Norway, Iceland, Faroe Islands, Russia and Alaska, the distant water pelagic fleets of Japan and (potentially) the artisanal pelagic fleets in Brazil. The data reliability score for all of the aforementioned fisheries was 'Poor' (with the exception of the Alaskan demersal groundfish fleet, which was 'Medium'), indicating the need for further data as well as implementation of effective mitigation. While demersal fleets have some of the highest levels of bycatch, many pelagic fleets are also important due to the proportion of vulnerable albatrosses and Procellaria petrel species caught. Data gaps remain for a number of fleets (especially in the North Atlantic, Mediterranean and Pacific), and these urgently need to be addressed.

As most bycatch estimates, especially at regional and global scales, have considerable associated uncertainties, largely because of persistent fundamental deficiencies in data collecting and reporting, we cannot conclusively determine whether overall levels of seabird bycatch have increased or decreased in recent years. Nevertheless, there are a number of fisheries in which the overall level of estimated seabird bycatch has decreased significantly over the last decade. The single largest reported reduction is of ca. 67000 birds $\mathrm{yr}^{-1}$ in CCAMLR fisheries. Major reductions have also been reported in USA, South Africa and New Zealand fisheries, mainly stemming from the implementation of effective mitigation measures. Reductions are also thought to have occurred in the Japanese southern bluefin tuna fleet and pelagic fleets operating off Uruguay and Brazil, mainly due to reduced fishing effort and some implementation of mitigation measures. These fleets have historically caught large numbers of vulnerable albatross populations, indicating likely important reductions in the number of albatrosses being caught. However, this may still be insufficient to redress population declines if the proportion taken from diminishing populations has not also decreased.

Since Nel \& Taylor (2003), emerging bycatch problems have been identified in a number of fleets not previously documented, including the Spanish demersal fishery on Gran Sol (North Atlantic), the Namibian demersal longline fleet, the Russian demersal longline fishery in Kamchatka and the Sea of Okhotsk and (potentially) the artisanal pelagic longline fishery within the Brazilian EEZ. Some of these, such as the Namibian fleet, are also catching high proportions of Procellaria petrels and albatrosses. Others in the northern hemisphere are predominantly catching northern fulmars, shearwaters and gulls.

Furthermore, this paper takes no account of bycatch of seabird species (generally of very similar taxonomic composition) associated with trawl or gillnet fisheries, now recognised as contributing substantially to the global bycatch total, particularly in certain regions.

Previous studies have established that bycatch mortality for some seabird species (especially albatrosses and some petrels) is at levels that have potentially serious impacts, and in some cases are clearly unsustainable for known or likely source populations. Numerous seabird species are already globally threatened (sensu Red List Criteria of IUCN 2010), with longline interactions identified as the primary cause of many population declines. Continued bycatch mortality at current levels may well drive them to the brink of extinction.

Key recommendations emerging from this review follow:

(1) All relevant fisheries should implement, to minimum and consistent standards, systematic onboard observer programs to collect and report seabird bycatch information and should make such data available to all stakeholders in a timely and comprehensive fashion. There is an urgent need to collect bycatch data in those fisheries for which data are lacking or current data reliability is deemed 'Poor' (the latter includes 9 of the top 10 fleets identified in this review as having the highest levels of seabird bycatch globally). Regional fisheries management organisations have a key role to play in establishing such standards, notably by implementing the new FAO Best Practice Technical Guidelines for International Plan of ActionSeabirds (FAO 2008). Independently verifiable reduction in seabird bycatch should become one of the indicators of compliance with the UN Code of Conduct for Responsible Fisheries.

(2) Demersal fleets, particularly those in the Atlantic, account for some of the highest levels of current seabird bycatch. Considerable experience in other demersal fisheries indicates that such bycatch can be quickly and substantially reduced (at minimal cost to the fisheries concerned) to levels that pose a negligible threat to populations. The mandatory use of best-practice mitigation measures for the fisheries involved, using only measures of proven efficacy, should urgently be implemented in these fisheries.

(3) Seabird bycatch in a number of pelagic fleets is particularly significant due to the proportion of threatened albatrosses and Procellaria petrels being caught. This review has demonstrated substantial reductions in bycatch in some key pelagic fisheries. 
Nevertheless, mitigation measures for pelagic fisheries are less well established than those for demersal fleets: some research is underway and more is needed to improve the design of measures such as line weighting and streamers lines. Additional research is needed to facilitate uptake of mitigation measures in these fisheries and to monitor the effectiveness of implementation with a view to adaptive management.

Acknowledgements. We thank E. Howgate for work on the initial collation and analysis of some of the data presented here. Thanks also to S. Cranwell, E. Frere, K. Hackwell, B. Heneman, B. Lascelles, F. Rabuffetti, I. Ramírez, G. Robertson, B. Watkins and S. Waugh for their input to earlier drafts. Following an initial review of the data, country-by-country summaries were sent to relevant experts for verification. We thank the following for their input: J. Andrade, P. Arcos, B. Baker, Á. Barros, L. Bugoni, S. Christensen-Dalsgaard, S. Crofts, A. Domingo, L. Enriquez, E. Frere, S. GarcíaBarcelona, M. Kiyota, K. Knowles, S. Løkkeborg, J. Mangel, C. Moreno, G. Munro, T. Neves, B. Olsen, J. Pearce, S. Petersen, I. Ramírez, K. Rivera, J. Alfaro Shigueto, SEO/ BirdLife, G. Tuck and R. Wanless. We particularly thank the 4 anonymous reviewers for their constructive and helpful comments.

\section{LITERATURE CITED}

Abraham ER, Thompson FN (2009) Capture of protected species in New Zealand trawl and longline fisheries, 1998-99 to 2006-07. Draft New Zealand Aquatic Environment and Biodiversity Report No 24. New Zealand Department of Conservation, Wellington

ACAP (Agreement on the Conservation of Albatrosses and Petrels) (2009) Species assessments. Available at www. acap.aq/acap-species (accessed 20 Nov 2009)

AFMA (Australian Fisheries Management Authority) (2007) Western tuna and billfish fishery. Six-monthly bycatch action plan (BAP) progress report, March 2007. AFMA, Canberrra (cited in Baker \& Finley 2008) Available at www.afma.gov.au/information/publications/fishery/baps/ docs_reports/bap_imp_rep_wtbf_200703.pdf (accessed 16 May 2008)

Anon. (2001) Final United States national plan of action for reducing the incidental catch of seabirds in longline fisheries. US Department of Commerce, National Oceanic and Atmospheric Administration (NOAA) and National Marine Fisheries Service (NMFS), Silver Spring, MD. Available at www.fakr.noaa.gov/protectedresources/ seabirds/npoa/npoapdf (Accessed 20 Nov 2009)

Anon. (2004) National plan of action to reduce the incidental catch of seabirds in New Zealand fisheries. Ministry of Fisheries (MoF) and Department of Conservation (DoC), Wellington. Available at www.doc.govt.nz/upload/documents/conservation/native-animals/birds/npoa.pdf (accessed 20 Nov 2009)

Anon. (2008) National plan of action for reducing the incidental catch of seabirds in longline fisheries - South Africa. Department of Environmental Affairs and Tourism, South Africa. Available at ftp://ftp.fao.org/FI/DOCUMENT/ IPOAS/national/southafrica/NPOA-Seabirds.pdf (Accessed 20 Nov 2009)
Ariz J, Delgado de Molina A, Ramos ML, Santana JC (2006) Checklist and catch rate data by hook type and bait for bycatch species caught by Spanish experimental longline cruises in the south-western Indian Ocean during 2005. IOTC-2006-WPBy-04

Arnold JM, Brault S, Croxall JP (2006) Albatross populations in peril? A population trajectory for black-browed albatrosses at South Georgia. Ecol Appl 16:419-432

Artyukhin YB, Vinnikov AV, Terentiev DA (2006) Seabirds and bottom longline fishery in the Kamchatka region. Environmental impacts of fisheries. World Wildlife Fund (WWF) Russia, Moscow

Ashford J (2002) Report of the WG-FSA inter-sessional subgroup on sampling catches from longliners. CCAMLR WG-FSA-02/52

Ayala L, Amoŕos S, Céspedes C (2008) Catch and bycatch of albatross and petrel in longline and gillnet fisheries in northern Peru. Final report to the Rufford Small Grants for Nature Conservation. Apeco. Available at www. ruffordsmallgrants.org/files/Progress \% 20Report_O.doc (Accessed 20 Nov 2009)

Baker GB, Finley LA (2008) National assessment report for reducing the incidental catch of seabirds in longline fisheries (2008 assessment report). Report to Australian Government Department of Agriculture, Fisheries and Forestry, Canberra

Baker GB, Double MC, Gales R, Tuck GN and others (2007) A global assessment of the impact of fisheries-related mortality on shy and white-capped albatrosses: conservation implications. Biol Conserv 137:319-333

- Barbraud C, Delord K, Marteau C, Weimerskirch H (2009) Estimates of population size of white-chinned petrels and grey petrels at Kerguelen Islands and sensitivity to fisheries. Anim Conserv 12:258-265

Bartle AJ (1991) Incidental capture of seabirds in the New Zealand and sub-Antarctic squid trawl fishery, 1990. Bird Conserv Int 1:351-359

> Belda EJ, Sanchez A (2001) Seabird mortality on longline fisheries in the western Mediterranean: factors affecting bycatch and proposed mitigating measures. Biol Conserv 98:357-363

BirdLife International (2004) Tracking ocean wanderers: the global distribution of albatrosses and petrels. Results from the Global Procellariiform Tracking Workshop, 1-5 September 2003, Gordon's Bay, South Africa. BirdLife International, Cambridge

BirdLife International, ACAP (2009) Seabird bycatch mitigation factsheets. BirdLife International, Cambridge. Available at www.rspb.org.uk/ourwork/policy/marine/international/advocacy/mitigationfactsheets.aspx (Accessed 15 Mar 2010)

Brothers NP (1991) Albatross mortality and associated bait loss in the Japanese longline fishery in the Southern Ocean. Biol Conserv 55:255-268

Brothers NP (2008) How accurate are observer reported kills of albatrosses on longlines? Final Report - October 2008. Duke University and Blue Ocean Institute, Durham, NC. Available at http://bycatch.env.duke.edu/publicationsandreports/Brothers2008.pdf (Accessed 20 Nov 2009)

Brothers N, Cooper JP, Løkkeborg S (1999) The incidental catch of seabirds by longline fisheries: worldwide review and technical guidelines for mitigation. FAO Fisheries Circular No 937. FAO, Rome

> Bugoni L, Mancini PL, Monteiro DS, Nascimento L, Neves TS (2008a) Seabird bycatch in the Brazilian pelagic longline fishery and a review of capture rates in the southwestern Atlantic Ocean. Endang Species Res 5:137-147 
Bugoni L, Neves TS, Leite NO Jr, Carvalho D and others (2008b) Potential bycatch of seabirds and turtles in hookand-line fisheries of the Itaipava Fleet, Brazil. Fish Res 90: 217-224

Bull LS (2007) Reducing seabird bycatch in longline, trawl and gillnet fisheries. Fish Fish 8:31-56

CCAMLR (Commission for the Conservation of Antarctic Marine Living Resources) (2008) 27th Scientific Committee Meeting for the Commission for the Conservation of Antarctic Marine Living Resources (SC-CCAMLR) Report. Annex 6: Report of the ad hoc working group on incidental mortality associated with fishing, 13-17 October 2008, Hobart, Tasmania

Clemens A (2006) Annual report on seabird interactions and mitigation efforts in the Hawaii longline fishery for 2005. National Marine Fisheries Service Report, Honolulu, HI

Cooper J, Baccetti N, Belda EJ, Borg JJ, Oro D, Papaconstantinou C, Sanchez A (2003) Seabird mortality from longline fishing in the Mediterranean Sea and Macaronesian waters: a review and a way forward. Sci Mar 67:57-64

Crawford RJM, Shelton PA (1978) Pelagic fish and sea bird interrelationships off the coasts of South West and South Africa. Biol Conserv 14:85-109

Crowder LB, Myers RA (2001) A comprehensive study of the ecological impacts of the worldwide pelagic longline industry. First Annual Report to the Pew Charitable Trusts, Philadelphia, PA. Available at www.ml.duke.edu/ faculty/crowder/research/crowder_and_myers_Mar_2002. pdf (Accessed 20 Nov 2009)

Croxall JP (2008) The role of science and advocacy in the conservation of Southern Ocean albatrosses at sea. Bird Conserv Int 18:S13-S29

Croxall JP, Rothery P (1991) Population regulation of seabirds: implications of their demography for conservation. In: Perrins CM, LeBreton JD, Hirons GM (eds) Bird population studies: relevance to conservation and management. Oxford University Press, Oxford, p 272-296

Croxall JP, Prince JP, Hunter I, McInnes S, Copestake PG (1984) The seabirds of the Antarctic Peninsula, islands of the Scotia Sea and Antarctic continent between $80^{\circ} \mathrm{W}$ and $20^{\circ} \mathrm{W}$ : their status and conservation. In: Croxall JP, Evans PGH, Schreiber RW (eds) Status and conservation of the world's seabirds. Proceedings of the ICBP Seabird Conservation Symposium, Aug 1982, Cambridge, p 637-666

Croxall JP, Prince PA, Rothery P, Wood AG (1998) Population changes in albatrosses at South Georgia. In: Robertson G, Gales R (eds) Albatross biology and conservation. Surrey Beatty and Sons, Chipping Norton, p 68-83

Cuthbert R, Hilton G, Ryan P, Tuck GN (2005) At-sea distribution of breeding Tristan albatrosses Diomedea dabbenena and potential interactions with pelagic longline fishing in the South Atlantic Ocean. Biol Conserv 121: 345-355

Dai X, Zhu J (2008) Species composition and size frequency data based on Chinese observer programme in central Pacific Ocean in 2008. 4th Regular Session of WCPFC, 11-22 Aug 2008, Port Moresby, Papua New Guinea. WCPFC-SC4-2008/EB-IP-9

Dai X, Xu L, Song L (2006) Observation of seabird bycatch in the Chinese longline fishery in the IATTC waters. 7 th Meeting of the Stock Assessment Working Group of IATTC, 15-19 May 2006, La Jolla, CA. SAR-7-05e

Dai X, Xu L, Song L (2008) Estimation of shark catch by the Chinese deep longline fishery in ICCAT waters. Collect Vol Sci Pap ICCAT 62(Suppl 5):1474-1476
DFO (Department of Fisheries and Oceans) Canada (2007) National plan of action for reducing the incidental catch of seabirds in longline fisheries. DFO Canada, Ottawa. Available at www.dfo-mpo.gc.ca/npoa-pan/npoa-pan/ npoa-seabirds-eng.htm (Accessed 20 Nov 2009)

Dietrich KS, Cornish VR, Rivera KS, Conant TA (2007) Best practices for the collection of longline data to facilitate research and analysis to reduce bycatch of protected species. Report of a workshop held at the International Fisheries Observer Conference, Sydney, Nov 8, 2004. NOAA Tech Memo NMFS-OPR-35

Dimech M, Darmanin M, Caruana R, Raine H (2008) Preliminary data on seabird bycatch from the Maltese longline fishery (central Mediterranean). Paper submitted to the ICCAT Standing Committee on Research and Statistics, 29 Sep-3 Oct, Madrid. SCRS/2008/027

Dunn E, Steel C (2001) The impact of longline fishing on seabirds in the northeast Atlantic: recommendations for reducing mortality. NOF Rapportserie Rep No. 5, The Royal Society for the Protection of Birds (RSPB), Sandy

FAO (Food and Agriculture Organization) (2008) Report of the expert consultation on Food and Agriculture Organization (FAO) best practice technical guidelines for IPOAseabirds. Bergen, Norway, 2-5 September 2008. FAO Fisheries and Aquaculture Rep No. 880. FAO, Rome. Available at ftp://ftp.fao.org/docrep/fao/011/i0459e/i0459 e00.pdf (Accessed 20 Nov 2009)

Favero M, Khatchikian CE, Aria A, Silva Rodriguez MP, Canete G, Mariano-Jelicich R (2003) Estimates of seabird by-catch along the Patagonian Shelf by Argentine longline fishing vessels, 1999-2001. Bird Conserv Int 13: 273-281

Gales R, Brothers N, Reid T (1998) Seabird mortality in the Japanese tuna longline fishery around Australia, 19881995. Biol Conserv 86:37-56

García-Barcelona S, Ortiz de Urbina JM, de la Serna JM, Alot E, Macías D (2009) Seabird bycatch in Spanish Mediterranean large pelagic longline fisheries, 2000-2008. International Commission for the Conservation of Atlantic Tuna (ICCAT) Meeting of the Standing Committee on Research and Statistics, 5-9 October 2009, Madrid. ICCAT-SCRS-2009-136

Goya E, Cardenas G (2003) Longline fisheries and seabirds in Peru. In: Løkkeborg S, Thiele W (eds) Report of the FAO/Birdlife South American workshop on implementation of NPOA-seabirds and conservation of albatrosses and petrels, Valdivia, 2-6 December 2003. FAO Fish Rep No. 751 FIIT/R751 (En). FAO, Rome, p 14-15. Available at ftp://ftp.fao.org/docrep/fao/007/y5742e/y5742e00.pdf (Accessed 20 Nov 2009)

Hata DN (2006) Incidental captures of seabirds in the US Atlantic pelagic longline fishery, 1986-2005. Report to NOAA Fisheries, Southeast Fisheries Science Center, Protected Resources and Biodiversity Division, Miami, FL

Heery E, Bellman MA, Majewski J (2010) Estimated bycatch of marine mammals, seabirds, and sea turtles in the 2002-2008 U.S. west coast commercial groundfish fishery. West Coast Groundfish Observer Program, NWFSC, Seattle, WA

Huang HW, Chang KY, Tai JP (2008a) The impact of Taiwanese longline fisheries on seabirds in the Atlantic Ocean. Paper submitted to the 2008 meeting of the SubCommittee on Ecosystems and Bycatch, Madrid, March 10-14, 2008. ICCAT SCRS-2008-030

Huang HW, Chang KY, Tai JP (2008b) Overview of the interaction between seabird and Taiwanese longline fisheries in the Pacific Ocean. 4th Regular Session of the Scientific 
Committee of WCPFC, 11-22 August 2008, Port Moresby, Papua New Guinea. WCPFC-SC4-2008/EB-WP-5

Huang HW, Chang KY, Tai JP (2008c) Preliminary estimates of seabird bycatch of Taiwanese longline fisheries in the Indian Ocean. 4th Session of the Indian Ocean Tuna Commission (IOTC) Working Party on Ecosystems and Bycatch, 20-22 October 2008, Bangkok. IOTC-2008-WPEB-17

IATTC (Inter-American Tropical Tuna Commission) (2006) The fishery for tuna and billfishes in the Eastern Pacific Ocean in 2006. IATTC 75th Annual Commission Meeting, 25-29 June 2007, Cancun. IATTC-75-06. Available at www.iattc.org/PDFFiles2/IATTC-75-06-Tunas-and-billfishes-in-the-EPO-2006.pdf

ICCAT (International Commission for the Conservation of Atlantic Tunas) (2008) National fishing effort statistics reported to the ICCAT. Task II data. Available at www. iccat.int/en/t2ce.asp

ICES (International Council for the Exploration of the Seas) (2009) Overview on ecosystem, fisheries and their management in Icelandic waters. ICES North West Working Group (NWWG) Report. Available at www.ices.dk/reports/ ACOM/2009/NWWG/Sec\% 2007\%20Overview\%20n $\% 20$ ecosystem, $\% 20$ fisheries $\% 20$ and $\% 20$ their $\% 20$ management \%20in\%20Icelandic\%20waters.pdf (Accessed 20 Nov 2009)

Igual JM, Tavecchia G, Jenouvrier S, Forero MG, Oro D (2009) Buying years to extinction: Is compensatory mitigation for marine bycatch a sufficient conservation measure for long-lived seabirds? PLoS ONE 4:e4826

IUCN (International Union for the Conservation of Nature) (2010) The IUCN Red List of Threatened Species. Available at www.iucnredlist.org/apps/redlist/search (Accessed 15 Mar 2011)

Jimenez S, Domingo A, Brazeiro A (2009) Seabird bycatch in the Southwest Atlantic: interaction with the Uruguayan pelagic longline fishery. Polar Biol 32:187-196

Lawrence E, Giannini F, Crombie J (2009) Estimation of seabird bycatch rates across the eastern tuna and billfish fishery (ETBF). Australian Government Bureau of Rural Sciences, Canberra

Lawson T (2006) Scientific aspects of observer programmes for tuna fisheries in the western and central Pacific Ocean. Western and Central Pacific Fisheries Commission, 2nd Regular Session of the Scientific Committee, 13-24 August 2006, Manila. WCPFC-SC2-2006/ST WP-1

Lawson T (ed) (2007) Western and Central Pacific Fisheries Commission (WCPFC) - tuna fishery yearbook. SCP, Noumea, New Caledonia

Løkkeborg S (2003) Review and evaluation of three mitigation measures - bird-scaring line, underwater setting and line shooter - to reduce seabird bycatch in the north Atlantic longline fishery. Fish Res 60:11-16

Mejuto J, Garcia-Cortes B (2005) Documentacion sobre el preparacion de datos cientificos de la pesqueria Espanola de pez espada (Xiphias gladius) en las regiones del Pacifico, con especial referencia a los anos mas recientes 2002 y 2003. Inter-American Tropical Tuna Commission (IATTC) Data and Standards Review Meeting, 29-30 April 2005, La Jolla, CA. DC-1-02d

Mejuto J, Garcia-Cortes B, Ramos-Cartelle A, Ariz J (2007a) Preliminary overall estimations of bycatch landed by the Spanish surface longline fleet targeting swordfish (Xiphias gladius) in the Pacific Ocean and interaction with marine turtles and seabirds: years 1990-2005. 6th Meeting of the Inter-American Tropical Tuna Commission (IATTC) Bycatch Working Group, 9-10 February 2007, La Jolla, CA. BYC-6-INF A
Mejuto J, Garcia-Cortes B, de la Serna JM, Ramos-Cartelle A (2007b) Activity of the Spanish surface longline fleet catching swordfish (Xiphias gladius) in the Atlantic Ocean during the year 2005. International Commission for the Conservation of Atlantic Tuna (ICCAT) Meeting of the Standing Committee on Research and Statistics, Madrid. ICCAT SCRS-2007-110

Minami H, Hosono T, Kiyota M, Takeuchi Y (2009) Estimation of incidental takes of seabirds in the Japanese Southern Bluefin Tuna longline fishery in 2006-2007. 8th Meeting of the Commission for the Conservation of Southern Bluefin Tuna (CCSBT) Ecologically Related Species Working Group, 1-3 September 2009, Busan. CCSBT-ERS/ 0909/05

Moon DY, Kim SS, Koh JK (2005) A summary of the Korean tuna fishery observer program for the Pacific Ocean (2004-2005). Western and Central Pacific Fisheries Commission (WCPFC) Scientific Committee Meeting, 8-19 August 2005, Noumea, New Caledonia. WCPFCSC1 ST IP-3

Morant PD, Brooke RK, Abrams RW (1983) Recoveries in Southern Africa of seabirds breeding elsewhere. Ringing Migr 4:257-268

Moreno CA, Arata JA, Rubilar P, Hucke-Gaete M, Robertson G (2006) Artisanal longline fisheries in southern Chile: lessons to be learned to avoid incidental seabird mortality. Biol Conserv 127:27-36

Moreno CA, Vega R, Ruiz J, Flores H (2007) Albatross Task Force Chile-Activity Report, Apr-Sep 2007. Instituto de Ecología y Evolución, Universidad Austral de Chile, Santiago

Moreno CA, Castro R, Mujica LJ, Reyes P (2008) Significant conservation benefits obtained from the use of a new fishing gear in the Chilean Patagonian toothfish fishery. CCAMLR Science 15:79-91

MRAG (Marine Resources and Assessment Group) (2005) Illegal, unregulated and unreported (IUU) fishing on the high seas: impacts on ecosystems and future research needs. Final Report, August 2005. MRAG Ltd, London

Nel DC, Taylor FE (2003) Globally threatened seabirds at risk from longline fishing: international conservation responsibilities. BirdLife International Seabird Conservation Programme, BirdLife South Africa, Cape Town. Available at www.birdlife.org/action/campaigns/save_the_albatross/ fao_doc3.pdf (Accessed 20 Nov 2009)

NOAA (2006) Annual seabird bycatch estimates for 2006. Alaska Fisheries Science Center - Seabird Coordinated Studies, Juneau, AK, NOAA. Available at www.afsc.noaa. gov/refm/reem/doc/Alaska_2006seabirdbycatch.pdf (Accessed 20 Nov 2009)

Petersen SL (2008) Understanding and mitigating vulnerable bycatch in Southern African longline and trawl fisheries. $\mathrm{PhD}$ thesis, University of Cape Town

Petersen SL, Honig MB, Nel DC (2007) The impact of longline fisheries on seabirds in the Benguela Current large marine ecosystem. In: Petersen S, Nel D, Omardien A (eds) Towards an ecosystem approach to longline fisheries in the Benguela: an assessment of impacts on seabirds, sea turtles and sharks. World Wildlife Fund (WWF) South Africa Report Series - 2007/Marine/001

Phillips RA, Tuck GN, Wood AG, Croxall JP (2010) Longline fisheries and the decline of the wandering albatross at South Georgia. First World Seabird ConferenceAbstracts of Invited Presentations, 7-11 Sept 2010, Vancouver, BC, p 24. Available at https://www.confmanager. com/communities/c1813/files/hidden/docs/webdocs/Full_ Abstracts_Invited.pdf (Accessed 15 March 2011) 
Poncet S, Robertson G, Phillips RA, Lawton K, Phalan B, Trathan PN, Croxall JP (2006) Status and distribution of wandering, black-browed and grey-headed albatrosses breeding at South Georgia. Polar Biol 29:772-781

Pro Delphinus (2006) Assessment of seabird bycatch in Peruvian artisanal fisheries. Final Report to the British Petroleum Conservation Programme, Lima

Rivalan P, Barbraud C, Inchausti P, Weimerskirch H (2010) Combined impacts of longline fisheries and climate on the persistence of the Amsterdam albatross Diomedea amsterdamensis. Ibis 152:6-18

Rivera KS, Henry RW III, Shaffer SA, LeBoeuf N, VanFossen L (2008) Seabirds and fisheries in the IATTC area: an update. 9th Meeting of the Inter-American Tropical Tuna Commission (IATTC) Working Group on Stock Assessment, 12-16 May 2008, La Jolla, CA. IATTCSARM-9-11a

Robertson G, Gales R (eds) (1998) Albatross biology and conservation. Surrey Beatty and Sons, Chipping Norton

Ryan PG, Goren M, Petersen SL, Smith C (2009) Seabird bycatch on pelagic long-lines in the ICCAT area off South Africa in 2007 and 2008: the effect of individual vessel limits on bycatch rates. ICCAT Standing Committee on Research and Statistics, 5-9 October 2009, Madrid. SCRS/ 2009/086

Smith JL, Morgan KH (2005) An assessment of seabird bycatch in longline and net fisheries in British Columbia. Tech Rep Ser No. 401. Canadian Wildlife Service, Pacific and Yukon Region, Delta, BC

Sullivan BJ, Reid TA, Bugoni L (2006) Seabird mortality on

Editorial responsibility: Rory Wilson,

Swansea, UK factory trawlers in the Falkland Islands and beyond. Biol Conserv 131:495-504

Thompson RB, Phillips RA, Tuck GN (2009) Modeling the impact of fishery bycatch on wandering albatross of South Georgia: final results. Inter-sessional Meeting of the SCRS Sub-committee on Ecosystems, 8-12 June 2009, Recife. SCRS/2009/074

Tuck GS, Polacheck T, Croxall JP, Weimerskirch H (2001) Modeling the impact of fishery by-catches on albatross populations. J Appl Ecol 38:1182-1196

Valeiras J, Caminas JA (2003) The incidental capture of seabirds by Spanish drifting longline fisheries in the western Mediterranean Sea. Sci Mar 67:65-68

> Véran S, Gimenez O, Flint E, Kendall WL, Doherty PFJ, Lebreton JD (2007) Quantifying the impact of longline fisheries on adult survival in the black-footed albatross. J Appl Ecol 44:942-952

> Wanless RM, Ryan PG, Altwegg R, Angel A, Cooper J, Cuthbert R, Hilton GM (2009) From both sides: dire demographic consequences of carnivorous mice and longlining for the Critically Endangered Tristan albatross on Gough Island. Biol Conserv 142:1710-1718

Weimerskirch H, Capdeville D, Duhamel G (2000) Factors affecting the number and mortality of seabirds attending trawlers and long-liners in the Kerguelen area. Polar Biol 23:236-249

Xu L, Dai X, Song L (2007) Chinese tuna longline fishery in the Indian Ocean in 2006. 10th Session of the Indian Ocean Tuna Commission (IOTC) Scientific Committee, 5-9 Nov 2007, Victoria, Seychelles. IOTC-2007-SC-INF12

Submitted: April 29, 2010; Accepted: March 22, 2011

Proofs received from author(s): June 3, 2011 